\title{
Valoración de la incapacidad del osteosarcoma en pacientes en edad laboral
}

\author{
Valuation of the disability of the osteosarcoma \\ in patients in labour age
}

\author{
Susana Álvarez Gómez \\ Instituto Social de la Marina. Dirección Provincial de Madrid. España. \\ José Laureano González Pulido \\ Instituto Nacional de la Seguridad Social. Unidad Médica del Equipo de \\ Valoración de Incapacidades. Dirección Provincial. Granada. España. \\ Piedad López Roldán \\ Consejería de Sanidad. Madrid. España. \\ Recibido: 14-10-09 \\ Aceptado: 16-02-10 \\ CORRESPONDENCIA: \\ Piedad lópez Roldán \\ Centro de Especialidades Jaime Vera \\ Avda. de España s/n \\ 28220 Coslada (Madrid). España. \\ Tfno.: 916694254 \\ E-mail: plopez.ims@salud.madrid.org
}

Resumen

Si bien los tumores óseos malignos son poco frecuentes, implican un mal pronóstico y requieren tratamientos muy agresivos que repercuten en la calidad de vida de los pacientes afectados y desde luego también en su actividad laboral. De hecho, un alto porcentaje de pacientes finalmente obtienen una incapacidad permanente. De todos los tumores óseos malignos, destaca el osteosarcoma que constituye el 30-40\% de los mismos. Con este trabajo se pretenden analizar los factores que el médico evaluador, adscrito a un equipo de valoración de incapacidades, debe considerar para delimitar si el paciente puede o no continuar con su actividad laboral tras el diagnóstico y tratamiento de un osteosarcoma. En dicha valoración funcional se deberían incluir los requerimientos profesionales recogidos en la guía de valoración profesional del Instituto Nacional de la Seguridad Social (INSS) y las escalas mas utilizadas actualmente para la valoración funcional.

Med Segur Trab (Internet) 2010; 56 (218): 85-92

Palabras Clave: osteosarcoma, incapacidad, requerimientos profesionales, escalas funcionales.

Abstract

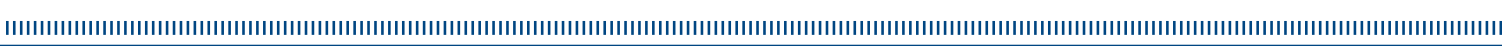

Though the malignant bone tumors are slightly frequent, they imply a poor prognosis and require aggressive treatments that affect the quality of life of patients and also in labour activity of course of course. In fact, a high percentage of patients finally obtain a permanent disability. Of all the malignant bone tumors, stands out the osteosarcoma that constitutes $30-40 \%$ of all of them. This work aims to analyze the factors that the medical assessor, assigned to a disability assessment team, should consider to define whether the patient may or may not continue its labour activity after the diagnosis and treatment of osteosarcoma. In the above mentioned functional assessment should include professional requirements (listed in the guide of professional assessment of Instituto Nacional de la Seguridad Social (INSS) and the scales currently used for functional assessment.

Med Segur Trab (Internet) 2010; 56 (218): 85-92

Key words: Osteosarcoma, disability, professional requirements, functional scales. 


\section{INTRODUCCIÓN}

Si bien los tumores malignos óseos son poco frecuentes, ya que no superan el 0.2 $0.5 \%$ de todos los cánceres, su importancia radica en que afectan a pacientes jóvenes en la segunda década de la vida, implican mal pronóstico en su evolución natural y suelen requerir tratamientos radicales, muy agresivos y sofisticados (1). Sin embargo, en los últimos años se ha producido un enorme progreso en el conocimiento de su biología, historia natural y métodos diagnósticos que, junto con los tratamientos, están suponiendo mayores posibilidades de curación. Y todo ello, sin duda repercute en la actividad laboral de los pacientes con esta patología.

Por ello, a través de este artículo, queremos proponer al médico evaluador una metodología para la valoración de pacientes con osteosarcoma, así como presentarle una serie de escalas de valoración funcional.

\section{Osteosarcoma}

Los tumores óseos se clasifican según las características celulares tumorales y sus productos; así, se distinguen lesiones que producen osteoide (osteosarcoma), colágeno (histiocitoma fibroso maligno), cartílago (condrosarcoma) y lesiones vasculares o de origen no bien determinado (sarcoma de Ewing, entre otros).

El osteosarcoma, o tumor osteogénico, constituye entre el 30-40\% de los tumores óseos malignos. Formado por células en huso de origen mesenquimatoso y con aspecto sarcomatoso, presenta un predominio en el sexo masculino $(1,5: 1)$, aparece en la segunda década de la vida (70-75\% de los casos) con un segundo pico en la sexta década de la vida, por lo que presenta una distribución por edades bimodal (2). La incidencia es de 1 a 3 casos por millón de habitantes.

\section{Repercusión en la aptitud laboral}

La valoración de la capacidad laboral de un trabajador requiere objetivar las limitaciones orgánicas y/o funcionales que una lesión o una enfermedad haya originado en el trabajador, pero también resulta imprescindible conocer las competencias y tareas realizadas y los requerimientos del puesto de trabajo, con objeto de poder determinar si las limitaciones impiden al trabajador desarrollar su actividad laboral. Por ello, La Legislación Española en materia de valoración de incapacidades en el Sistema de Seguridad Social establece que quede determinada la profesión del trabajador en las distintas modalidades de la calificación de la incapacidad.

Desde el punto de vista de la valoración de la incapacidad temporal y permanente de los pacientes con osteosarcoma, al igual que en otros pacientes oncológicos, lo primero que se debe tener en cuenta es la necesidad de un tratamiento oncológico agresivo, como es la cirugía, quimioterapia y radioterapia, que implica una incapacidad temporal durante su aplicación, debido a los efectos secundarios derivados del mismo.

Los osteosarcomas en estadio avanzado y de mal pronóstico suelen precisar tratamientos oncológicos de larga duración; la reincorporación laboral en estos casos no debe plantearse hasta finalizar el tratamiento de los mismos y haber realizado el primer estudio de extensión, el cual suele hacerse en la práctica clínica a los tres meses de finalizar el tratamiento. La incapacidad temporal se prolongará el tiempo necesario, no siendo infrecuente agotar el período máximo (3). 


\section{PROCEDIMIENTOS DE EVALUACIÓN}

\section{Comprobaciones del medico evaluador}

Para el control de la incapacidad temporal o, en su caso, para la valoración de la incapacidad permanente de un paciente con osteosarcoma, proponemos que el médico evaluador designado a tal fin, valore los siguientes epígrafes:

Exploraciones a realizar y datos a obtener de las mismas

Factores Pronósticos

Factores Psicológicos

\section{Exploraciones a realizar y datos a obtener de las mismas}

VALORACIÓN DEL TUMOR EN SÍ Y DE LOS DEFECTOS QUE CAUSA EN EL ÓRGANO AFECTADO:

En el tratamiento quirúrgico del osteosarcoma, hay dos tipos de procedimientos: los de preservación del miembro o las amputaciones.

En los procedimientos de preservación del miembro, en ocasiones, cuando está afectado un hueso prescindible (por ejemplo, el peroné, la costilla, la escápula, la porción distal del cúbito o el ala del ilion), no se requiere reconstrucción. Pero todos los tipos de reconstrucciones que preservan el miembro exigen modificaciones del estilo de vida (evitar actividades con excesiva carga de impacto, tales como correr y saltar) (4). En el caso de artrodesis, la fusión de la articulación después de la resección del tumor se puede efectuar mediante injertos óseos autólogos y dispositivos de fijación interna. Una vez consolidada, la extremidad es tan durable como un miembro normal, pero este procedimiento presenta complicaciones de pérdida de movimiento, un período de consolidación prolongado y fracturas tardías. Estos problemas aumentan con la quimioterapia y la irradiación postoperatorias (4).

En el caso de reemplazos con aloinjerto, al igual que en las artrodesis, puede haber un período de consolidación prolongado, fracturas tardías o colapso subcondral, que exigen el reemplazo articular total.

En el caso de reemplazo endoprotésico a medida, se preserva la función, la estética y la longitud del miembro y requiere un breve tiempo de consolidación, que no se ve afectado por la terapia coadyuvante postoperatoria. Así, la rehabilitación puede ser precoz. Sin embargo, se desconoce la durabilidad de la fijación y existe el riesgo de fractura por fatiga del metal, aunque las prótesis pueden ser revisadas sin dificultad en caso de que sobrevengan problemas (3).

\section{VALORACIÓN DE LOS FACTORES RELACIONADOS CON EL PROPIO PACIENTE}

Estado general del paciente o capacidad de valerse por sí mismo. Nos lo aportará la aplicación de las escalas más utilizadas, como son las de Karnofsky (Tabla I) y ECOG (Eastern Cooperative Oncology Group) (Tabla II) (3).

\section{VALORACIÓN DE LAS SECUELAS DERIVADAS DE LOS PRINCIPALES TRATAMIENTOS} ONCOLÓGICOS

Secuelas de la cirugía del tumor primario

- Principales amputaciones en el miembro inferior:

- Amputación transmetatarsiana: no se requerirá una prótesis especial, basta con rellenar el hueco del zapato con una almohadilla.

- Amputación de Syme (amputa tibia y peroné a nivel de la articulación del tobillo, por encima de los maleolos) permite conservar la longitud del miembro inferior y la conservación de un punto de apoyo vivo. Las prótesis han mejorado y permiten un buen encaje. La marcha puede realizarse con una simple copa con un tacón adaptado. También puede practicarse una prótesis con pie adaptado, lo que proporciona un mejor resultado estético; es muy fácil de poner y quitar y permite realizar un trabajo pesado sin problemas. 
- Amputación por debajo de la rodilla: la prótesis habitual es de contacto con apoyo en tendón rotuliano y cóndilos tibiales. El paciente puede salir andando del hospital en un 80\% de los casos en 4-5 semanas.

- Amputación por encima de la rodilla: la rehabilitación es difícil y las probabilidades de conseguir la marcha son menores que en los casos anteriores, teniendo que usar una silla de ruedas.

- Principales amputaciones en miembro superior:

La sustitución protésica es menos satisfactoria que en el miembro inferior, fundamentalmente por la complejidad de las funciones que realiza este miembro. Los dispositivos protésicos sólo son capaces de realizar algunos movimientos muy simples. Así, existe la llamada prótesis de trabajo, que puede realizar función de pinza y gancho, aunque es antiestética. En la prótesis funcional, el funcionamiento es desencadenado por un estímulo muscular del muñón de amputación, que origina la puesta en marcha de un dispositivo eléctrico o neumático, que, a su vez, activa la prótesis. La prótesis estética trata de imitar -del modo mas perfecto- la parte del miembro amputada. La utilidad viene dada por la movilidad de las articulaciones reales existentes. Así:

- Amputación en la muñeca: conserva la pronación del antebrazo, es útil para la acción de presión y sostén, incluso sin prótesis, y precisa de una prótesis corta.

- Amputación a través del antebrazo: si es por el tercio inferior, permite un buen encaje con el tipo de prótesis de la desarticulación de la muñeca, así como un aceptable movimiento de pronosupinación del antebrazo. El mejor nivel lo constituye el tercio medio. La amputación superior, dejando un muñón corto del antebrazo, es superior a la desarticulación del codo. Se entiende por muñón corto aquél que es inferior al 30\% de la longitud del antebrazo. Este muñón tan corto plantea gran dificultad para el implante de una prótesis, así como para la flexión de la articulación que permita al extremo de la prótesis alcanzar la boca o la cara.

- Desarticulación del codo: tiene una clara ventaja sobre la amputación del brazo, ya que la paleta del húmero permite un buen encaje en la prótesis adecuada y la rotación del húmero puede transmitirse a la prótesis con estabilidad.

- Amputación del brazo: hay que procurar que la colocación de la prótesis permita tener el codo al mismo nivel que en el lado contralateral, por lo que, teniendo en cuenta el encaje de aquella, debe seccionarse el hueso unos $4 \mathrm{~cm}$. por encima de la articulación.

La amputación alta por encima del pliegue axilar tiene un escaso valor funcional; sin embargo, el conservar la cabeza humeral proporciona un valor más estético.

En definitiva, el nivel de amputación es un tema capital en la valoración de todo amputado. En el caso que nos ocupa, donde las amputaciones más frecuentes van a ser en el miembro inferior, debemos tener en cuenta que la amputación por debajo de la rodilla supone un incremento del gasto energético de un $10 \%$, equivalente a una simple anquilosis del tobillo. La amputación por el muslo en pacientes de edad supone un incremento del 25 al 100\%. Este aumento de energía al andar viene dado por las oscilaciones del tronco al caminar. Una amputación transmetatarsiana bilateral permite una marcha correcta con una prótesis de pie. Una amputación bilateral de ambos muslos supone una reducción del paciente a una silla de ruedas en más del 80\% de los casos. Una amputación por debajo de la rodilla de un lado y por el muslo contralateral tiene un 30\% de probabilidad de caminar con prótesis (5).

Por tanto, la capacidad de adaptación al trabajo o la necesidad de cambiar de profesión van a depender del déficit producido (amputación alta o baja), de la profesión previa (ejercicio físico, habilidad, aspecto estético), de la voluntad del sujeto y de la habilidad de los médicos encargados de la rehabilitación (16). 
- Patología del muñón de la operación

Habrá que valorar la posible existencia de un muñón defectuoso (por defectos cutáneos, óseos, de partes blandas o articulares) o de un muñón doloroso (con las siguientes formas clínicas: neuroma doloroso, miembro fantasma doloroso o causalgia) (5).

— Evaluación de las Amputaciones: Escala de Mélennec (miembro inferior).

- Amputación de ambos muslos: $75 \%$

- Amputación de ambas piernas: $60 \%$

- Amputación a nivel de cadera: $60 \%$

- Amputación a nivel de muslo: $50 \%$

- Amputación a nivel de rodilla: $40 \%$

- Amputación a nivel de pierna: $30-35 \%$

- Amputación a nivel de antepié: $25 \%$

- Amputación transmetatarsiana: 15\%

- Amputación de todos los dedos: $10 \%$

- Amputación del dedo gordo: 5\%

- Amputación de un dedo del pie: 2\%

Secuelas de la quimioterapia

La quimioterapia puede originar toxicidad inmediata, precoz (días o semanas tras el tratamiento), retardada (semanas o meses después del tratamiento) y tardía (meses o años). Es frecuente la toxicidad a nivel digestivo (náuseas, vómitos, diarreas, mucositis), a nivel hepático, renal, hematológico, dermatológico, ocular y neurológico. Toxicidad toda ella, generalmente precoz o inmediata y que suele revertir (3).

Sin embargo, con el metotrexate o la bleomicina se ha descrito la aparición de una posible fibrosis pulmonar. En estos casos, las pruebas funcionales respiratorias revelarán una disminución de la capacidad de difusión y un defecto ventilatorio restrictivo (4). A nivel cardiológico, puede aparecer insuficiencia cardiaca congestiva y a nivel neurológico, neuropatías sensitivas. Estas secuelas generalmente no son reversibles y por sí mismas pueden ser origen de una incapacidad permanente (3).

Secuelas de la radioterapia

Las complicaciones específicas de la radioterapia dependen de la localización del tumor primario y del grado de irradiación. Los problemas mas comunes son: mala cicatrización de la herida o disminución de la amplitud del movimiento (4).

Secuelas del tratamiento quirúrgico de las metástasis pulmonares

Las resecciones pulmonares pueden dar lugar a trastornos ventilatorios restrictivos que se valorarán en función del grado de disnea, del déficit funcional encontrado en las pruebas funcionales respiratorias $y$ de la necesidad $o$ no de tratamiento con oxigenoterapia.

\section{Factores pronósticos}

Aunque la patología oncológica debe valorarse en función de las limitaciones derivadas del propio tumor o de las secuelas de las distintas terapias empleadas, es necesario tener en cuenta una serie de factores pronósticos que nos pueden dar una idea acerca de la evolución previsible de dicho proceso (6).

Los factores pronósticos más aceptados en el momento presente para el osteosarcoma medular de alto grado no metastático, son los siguientes:

- Relacionados con la respuesta a la quimioterapia: los pacientes con tumores del esqueleto axial, de sexo masculino y con una duración de los síntomas superior a 70 días son lo que presentan un menor índice de respuestas.

- Relacionados con la recidiva local: la cirugía incompleta o con márgenes afectos y la mala respuesta a la quimioterapia neoadyuvante son los dos factores de riesgo más importantes respecto a la progresión local. 
- Relacionados con la supervivencia global y libre de enfermedad: el principal es el grado de necrosis postquimioterapia. También son de interés la localización (peor en los tumores axiales), la presencia de metástasis, el tamaño (sobre todo, en tumores de las extremidades), la enfermedad residual postcirugía y los niveles de fosfatasa alcalina o LDH (7).

\section{Factores psicológicos}

Se han descrito alteraciones psicológicas en un 50\% de los pacientes con cáncer, predominando los síntomas depresivos (3).

En el caso del osteosarcoma también se han descrito alteraciones psicológicas asociadas a los cambios en la apariencia física, que constituyen otra fuente de estrés para estos pacientes. Su capacidad para aceptar y adaptarse a estos cambios repercute directamente en su estado emocional, en su calidad de vida y en su funcionamiento personal, familiar, social y laboral (8).

\section{REQUERIMIENTOS PROFESIONALES}

Con el objetivo de aportar una metodología de trabajo que permita identificar los requerimientos profesionales en la valoración de la capacidad laboral de los trabajadores, el Instituto Nacional de la Seguridad Social (INSS) ha elaborado la Guía de Valoración Profesional (actualmente se usa el borrador 1, versión de 4 de junio de 2008).

Se han confeccionado 150 fichas, correspondientes a las profesiones más frecuentes. Cada ficha contiene toda la información relativa a cada una de las profesiones. Se ha diseñado un sistema de valoración de requerimientos profesionales con 4 niveles de intensidad o exigencia, basado en criterios objetivos de medición e individualizados para cada uno de los requerimientos. En cada ficha se recogen estos apartados:

- Identificación de la profesión y descripción de competencias y tareas: se ha tomado como referencia para identificar cada profesión y realizar la descripción de competencias y tareas la Clasificación Nacional de Ocupaciones (CNO-94), aprobada mediante Real Decreto 917/1994, de 6 de mayo. Dado que esta clasificación es acorde con la Clasificación Internacional Uniforme de Ocupaciones (CIU0-88), también es homologable a las clasificaciones de países de nuestro entorno.

- Requerimientos profesionales: aptitudes o facultades psicofísicas que debe poseer un trabajador para realizar una profesión determinada. Para cada requerimiento existirá un nivel de intensidad o exigencia diferente en función de las características del trabajo a realizar.

La información se presenta en forma de tabla, donde se recoge para cada requerimiento el nivel de intensidad o exigencia del mismo, de menor a mayor, en una escala de 4 grados: 1 , baja; 2 , moderada; 3 , media-alta y 4 , muy alta.

Los requerimientos que se recogen son los siguientes:

- Carga física.

- Carga biomecánica (para columna, hombro, codo, mano, cadera, rodilla y tobillo/pie).

- Manejo de cargas.

- Trabajo de precisión.

- Sedestación, Bipedestación (estática y dinámica).

- Marcha por terreno irregular.

- Carga mental.

- Audición.

- Visión.

- Dependencia (falta de autonomía). 


\section{ESCALAS PARA LA VALORACIÓN FUNCIONAL}

La funcionabilidad o estado funcional es un concepto complejo que requiere al menos dos dimensiones para aproximarnos a su medida: La dimensión "estado funcional" y la dimensión "habilidad funcional". Así, la OMS define estado funcional como la realización de las actividades y tareas asociadas con el rol de vida ("an individual's performance of activities and tasks associated with life roles") y define la habilidad funcional como la capacidad actual o potencial para realizar las actividades y tareas que se esperan normalmente de los individuos ("an individual's performance of activities and tasks associated with life roles").

Existen diferentes índices o escalas que permiten al examinador conocer la situación funcional de un paciente concreto. En nuestro caso, nos interesan aquellos índices o escalas que se fijan en pacientes oncológicos y/o amputados, ya que el paciente diagnosticado de osteosarcoma es un paciente oncológico y en muchos casos, debido a la localización del tumor y el tratamiento realizado es también un paciente amputado. Existen diferentes escalas de valoración funcional en el paciente amputado, entre ellas, la Clasificación de Pohjolainen, la Escala de Volpicelli, la Clasificación de Russek, el Instrumento de Houghton, el Indice de Capacidad Motriz, el Cuestionario de Evaluación Protésica, el Cuestionario SAT-PRO, y el Indice MSTS.

De todas las escalas, destaca el Indice MSTS o de Enneking, que pretende hacer una evaluación clínica que permite comparar la función física resultante de los distintos tratamientos quirúrgicos en pacientes con sarcoma en extremidades y que se utiliza para miembros adaptables a amputados. Este cuestionario es cumplimentado por el médico, por lo que los resultados obtenidos, aun siendo importantes, no muestran una consistente relación con la funcionalidad del paciente (9). Otro índice específico para amputados es el cuestionario SAT-PRO siempre que utilicen algún tipo de prótesis, ya que todas las cuestiones que debe responder el paciente versan sobre la adaptación a la prótesis. Otro índice útil para pacientes amputados es el Instrumento de Houghton que incluye preguntas sencillas sobre la capacidad funcional y adaptación a la prótesis. Es muy utilizado en nuestro medio (10).

Como índices de valoración de la vida diaria se utilizan el Índice de Barthel, que se centra en el cuidado personal y en la movilidad, y lo realiza el profesional o el cuidador directo, el Edinburg Rehabilitation Status Scale o ERSS validada para evaluación de personas con discapacidad que viven en comunidad, el índice ADL y el RNLI. En la Tabla III se recogen las características de los índices mas empleados.

\section{DETERMINACIÓN DEL TIPO DE CONTINGENCIA PROTEGIDA}

En cuanto a la contingencia determinante de la incapacidad en el osteosarcoma, conviene reseñar que, según el Decreto 1995/1978, de 12 de mayo de 1978, por el que se aprobaba el Cuadro de Enfermedades Profesionales, se reconocía el cáncer de hueso producido por radiaciones ionizantes como enfermedad profesional en todos los trabajos expuestos a la acción de los rayos $\mathrm{X}$ o de las sustancias radioactivas naturales o artificiales o a cualquier fuente de emisión corpuscular.

Sin embargo, en el Anexo 1 del R.D. 1299/2006, de 10 de noviembre, por el que se aprueba el nuevo Cuadro de Enfermedades Profesionales, vigente desde 1 de enero de 2007, ha desaparecido el osteosarcoma como enfermedad profesional producida por radiaciones ionizantes. 


\section{CONCLUSIÓN}

La valoración médica de un paciente con osteosarcoma debe integrar:

- Los efectos del proceso y del tratamiento, tanto en la aptitud laboral (para lo que se hace necesario el conocimiento de las exigencias profesionales) como en la vida cotidiana.

- Siempre la calidad del paciente oncológico y eventualmente como paciente amputado.

— La valoración profesional y la percepción por el paciente de su calidad de vida.

\section{BIBLIOGRAFÍA}

1. Skubitz KM., D’Adamo D. Sarcoma. Mayo Clin Proc. 2007; 82:1409-1432.

2. Gaspar C., Guillem V. Tumores Óseos Malignos. En: Oncología Médica. Diaz-Rubio E., Guillem Porta V. 1998. pag. 161-169.

3. Criterios orientativos para la valoración médica de la incapacidad en patologías oncológicas. Manual de Actuación de Médicos del INSS. 2003.

4. Chow G., Eckardt J. Procesos malignos del hueso. En: Cameron R. Oncología Práctica. Buenos Aires. Editorial Médica Panamericana. 1995, pág, 443-454.

5. González F. Amputaciones y desarticulaciones. En Durán H. Arcelus I.M., García-Sancho L., González F., Álvarez J., Fernández L., Méndez J. Cirugía. Tratado de Patología y Clínica Quirúrgicas vol. 3. $2^{\mathrm{a}}$ Edición. Madrid. Mac Graw-Hill. Interamericana de España. 1996 pag. 5122-5143.

6. Kempf-Bielack B, Bielack SS, Jürgens H, et al.: Osteosarcoma relapse after combined modality therapy: an analysis of unselected patients in the Cooperative Osteosarcoma Study Group (COSS). J Clin Oncol 2005; 23 (3): 559-568.

7. Martínez B., Espinosa E., Zamora P. Osteosarcoma. Factores pronósticos para la supervivencia y la recaída. En: González M. Factores pronósticos en Oncología. 2a Edición. Madrid. Mc Graw-Hill Interamericana de España. 2005, pág. 224-233.

8. Fernández A. Asociación Española Contra el Cáncer. Facultad de Psicología. Universidad Complutense de Madrid. Psicooncología. 2004; Vol.1, (núms. 2-3):169-180.

9. Davis A.M., Wright J.G., Williams J.I. et al. Development of a measure of physical function for patients with bone and soft tissue sarcoma. Quality of Life Research 1996; 5:508-516.

10. Mencías Hurtado A.B., Suárez Alonso F., Hernández Kauffman M.R., et al. Valoración de la capacidad funcional del paciente amputado tras un año de protetización. Avances Traum 2006; 36(4):251-253.

\section{AGRADECIMIENTOS}

Queremos expresar nuestro agradecimiento al Doctor D. Raúl J. Regal Ramos, médico evaluador del INSS en la Unidad Médica del Equipo de Valoración de Incapacidades de la Dirección Provincial de Madrid por su colaboración, total disponibilidad y sus siempre oportunas aportaciones en la tutoría de este estudio y a la Doctora Josefa Ruiz Figueroa, Jefa de Estudios del Diploma de Valoración Médica de Incapacidades de la Escuela Nacional de Medicina del Trabajo del Instituto de Salud Carlos III de Madrid, por su valiosa revisión y corrección de estilo. 